\title{
Viral Disease, Tissue Injury, Repair and Regeneration
}

\author{
Nicholas Ten ${ }^{1}$, Deisy Contreras ${ }^{1}$, Vidhya Kanagavel ${ }^{1}$ and Vaithilingaraja Arumugaswami ${ }^{1,2,3^{*}}$ \\ ${ }^{1}$ The Board of Governors Regenerative Medicine Institute, Cedars-Sinai Medical Center, Los Angeles, CA 90048, California, USA \\ ${ }^{2}$ Department of Surgery, Cedars-Sinai Medical Center, Los Angeles, CA 90048, California, USA \\ ${ }^{3}$ Department of Surgery, David Geffen School of Medicine, University of California at Los Angeles, Los Angeles CA 90095, California, USA
}

${ }^{*}$ Corresponding author: Vaithilingaraja Arumugaswami, MVSc., PhD, The Board of Governors Regenerative Medicine Institute, Cedars-Sinai Medical Center, Los Angeles, CA 90048, California, USA, Tel: +1 310248 8584; Fax: +1 310248 8066; E-mail: arumugaswami@cshs.org

Received date: July 08, 2014, Accepted date: November 20, 2014, Published date: November 27, 2014

Copyright: (C) 2014 Nicholas T et al. This is an open-access article distributed under the terms of the Creative Commons Attribution License, which permits unrestricted use, distribution, and reproduction in any medium, provided the original author(s) and source are credited.

\section{Abstract}

Adult stem cells present in various tissues play key roles in tissue repair and regeneration upon injury. The inflammatory responses associated with tissue damage that are caused by physical, chemical, infectious, nutritional and genetic factors activate stem cells to proliferate and differentiate. The severity and duration of the injury influence the outcome of tissue repair. Viral diseases are major public health problems and over 500 million people worldwide are affected with viral hepatitis. Virus infection of acute or chronic nature could disrupt the tissue homeostasis by altering cell function and architecture. Little is known about the effect of viral diseases on resident stem or progenitor cell population during tissue repair and the regeneration process. This review summarizes the liver-specific inflammatory and healing responses to injury and provides a detailed overview of the cellular and molecular basis of tissue regeneration following viral diseases. Understanding the behavior of resident stem or progenitor cells in response to tissue injury caused by infectious agents such as viruses can allow for the development of small molecule and cell-based therapy for tissue regeneration.

Keywords: Viral disease; Tissue injury; Adult stem cells; inflammatory responses.

\section{Introduction}

Living organisms evolved to repair or regenerate the injured tissues in order to enhance the rate of survival and fitness. Tissue injury is caused by physical, chemical, nutritional, genetic and infectious etiologies and, depending on their nature and severity, can lead to various healing responses at the cellular level. The scourge of viral disease outbreaks at pandemic and epidemic proportions shaped our evolution. Currently, over 500 million people are affected with viral hepatitis and an estimated 35.3 million are infected with human immunodeficiency virus (HIV) [1-4]. Besides, morbidity and mortality associated with seasonal influenza exert huge burden on the healthcare system and have tremendous impact on the economy [5]. Understanding host-pathogen interaction is crucial for counteracting viral diseases by development of vaccines and direct acting antiviral agents. Viral pathogens can be transmitted by person-to-person contact (aerosols, touch, contaminated blood and bodily fluids), food, water, arthropod vectors and inanimate objects. Upon entry to the host organism, viruses reach the target organs and establish localized or generalized infection.

Viral tissue tropism is determined by the cellular expression of viral entry receptors and host factors critical for completing the viral growth cycle. The liver-specific pathogen, hepatitis $\mathrm{C}$ virus (HCV) envelope proteins (E1, E2) have been shown to directly or indirectly interact with hepatocyte surface receptors including CD81, LDL receptors (LDL-R), scavenger receptor - B1 (SR-BI), and claudin1 [6]. The influenza virus hemagglutinin (HA) glycoprotein binds to cell membrane sialic acid for entry into upper respiratory tract cells [7]. Non-enveloped viruses, coxsackievirus B (CVB) and adenovirus-2/5 enter through the coxsackievirus and adenovirus receptor (CAR) $[8,9]$. Viral entry occurs through $\mathrm{pH}$-dependent or $\mathrm{pH}$-independent endocytosis pathway [10]. Cellular innate immune factors, such as toll-like receptors (TLRs), and retinoic acid inducible gene I (RIG-I) sense the viral pathogen associated molecular pattern (PAMP), which results in activation of IFN-alpha/beta pathways $[11,12]$. Inflammatory response and innate immune factors are key drivers for eliciting specific T- and B-cell mediated immune response.

Inflammation is the general response that occurs in many different organ systems in response to tissue/cell injury. It encompasses the combined effects of vascular changes and cellular reactions that have the collective purpose of removing the injurious stimuli and initiating the healing process [13]. Viral-mediated cell injury elicits acute and/or chronic inflammatory responses. Acute inflammation refers to inflammation of relatively short duration primarily characterized by the excretion of fluids and plasma proteins and the emigration of neutrophils to the site of injury by chemoattraction, which engulf necrotic tissue and pathogenic microorganisms [14-17]. Hepatitis A virus and influenza virus can cause acute infection, resulting in an acute inflammatory response $[18,19]$. Chronic inflammation, on the other hand, refers to inflammation of relatively long duration, and is characterized by the presence of macrophages and lymphocytes, and the extensive proliferation of blood vessels, tissue necrosis, and fibrosis/scar tissue formation [13]. Chronic disease can result from infection with hepatitis B virus, HCV, and herpes viruses (EpsteinBarr virus and cytomegalovirus). Depending on the nature of the viral pathogens, the infected cell's fate can be non-lytic, lytic, or cancerous transformation. Viral infection may cause tissue injury by triggering auto-immune responses. Pathogenesis of auto-immune diseases such as multiple sclerosis and type 1 diabetes mellitus has been linked to Epstein-Barr virus and enterovirus infections, respectively [20,21]. 
After inflammation, the healing process occurs either by regeneration, repair or a combination of both. Complete regeneration occurs in labile tissues that continuously divide such as the epithelia of the skin, gastrointestinal tract, and the hematopoietic system [22-24]. Viral enteritis caused by rotavirus, astrovirus and norovirus can be self-limiting and recovery of intestinal villus epithelial cells can occur [25-27]. Recovery and regeneration of upper respiratory tract lining epithelium takes place following common cold caused by rhinoviral infection [28]. Full functional regeneration can also occur in minor, non-chronic injuries to quiescent tissues such as that of the liver. Regeneration entails the proliferation of cells and tissues to functionally replace lost/damaged structures, and requires that the basement membranes of the extracellular matrix are intact. In cases in which permanent damage to the extracellular matrix is sustained, the repair process is initiated through the compensatory formation of nonfunctional scar tissue via collagen deposition. This occurs in chronic liver inflammation resulting in liver fibrosis/cirrhosis and in deep skin wounds $[23,29]$. As with many other processes in the body, our capacity for cellular regeneration declines with age. Understanding the complex interactions of viral infection and various host cells (inflammatory, immune and stem cells) at the systems biology level can provide new avenues to combat viral pathogens. Figure 1 presents the cell and molecular players involved in host-pathogen interaction. Though viral diseases affect various organs, we limit the scope of this review to the liver. The following sections focus on the stem cell niche, viral-mediated cell injury, and the repair and regeneration processes of the liver.

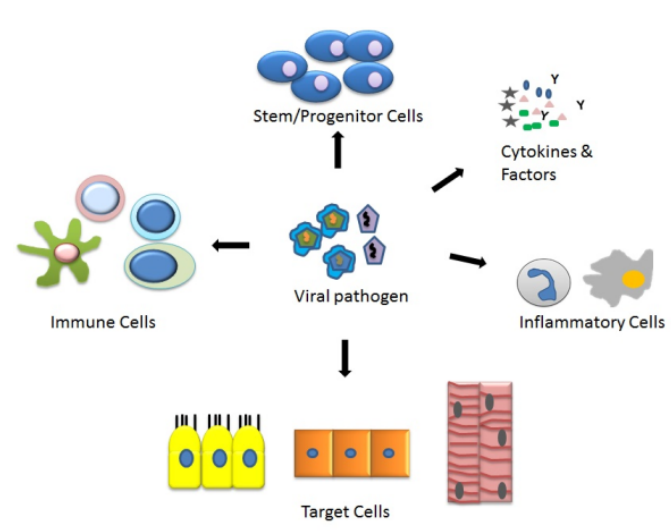

Figure 1: Schematic illustration of cell and molecular components involved in host-pathogen interaction. Viral-mediated injury to target cells elicits inflammatory response. Inflammatory cytokines and cells (neutrophils and macrophages) orchestrate the events of clearing dead cells, removal of pathogen and tissue remodeling which pave the way for healing by repair or regeneration. Tissuespecific stem and progenitor cells receive activation signals for proliferation and differentiation to become functional parenchymatous cells. Viral-specific immune response is mediated by antigen presenting cells, T- and B -lymphocytes.

\section{Hepatic Cellular Composition and Stem Cell Niche}

The liver, the largest metabolic organ, carries out both exocrine and endocrine functions. The functional compartments of the liver encompass the hepatic lobule and portal triad. The portal triad consists of the portal vein, hepatic artery and bile duct. From the portal triad, blood flows through the liver sinusoidal spaces where the nutrients, metabolites and waste products are transferred to the hepatic plates across the endothelial fenestrae (Figure 2). Sinusoidal blood drains into the central vein present in the hepatic lobule. Periportal and pericentral hepatocytes execute different but complementary metabolic roles depending on oxygen zonation. The parenchymal cells which make up approximately $70 \%$ of the whole liver are the hepatocytes which along with biliary epithelial cells, originate from embryonic endoderm. Moreover, the stromal cells, hepatic stellate cells (HSCs; Ito cells), Kupffer cells (resident macrophages) and sinusoidal endothelial cells that constitute the liver have a mesodermal - embryonic origin. Hepatic stellate cells reside in the space of Disse (space between hepatocytes and sinusoidal endothelium) in adult liver and are identified by the expression of desmin and glial fibrillary acidic protein in the quiescent state [30-32].

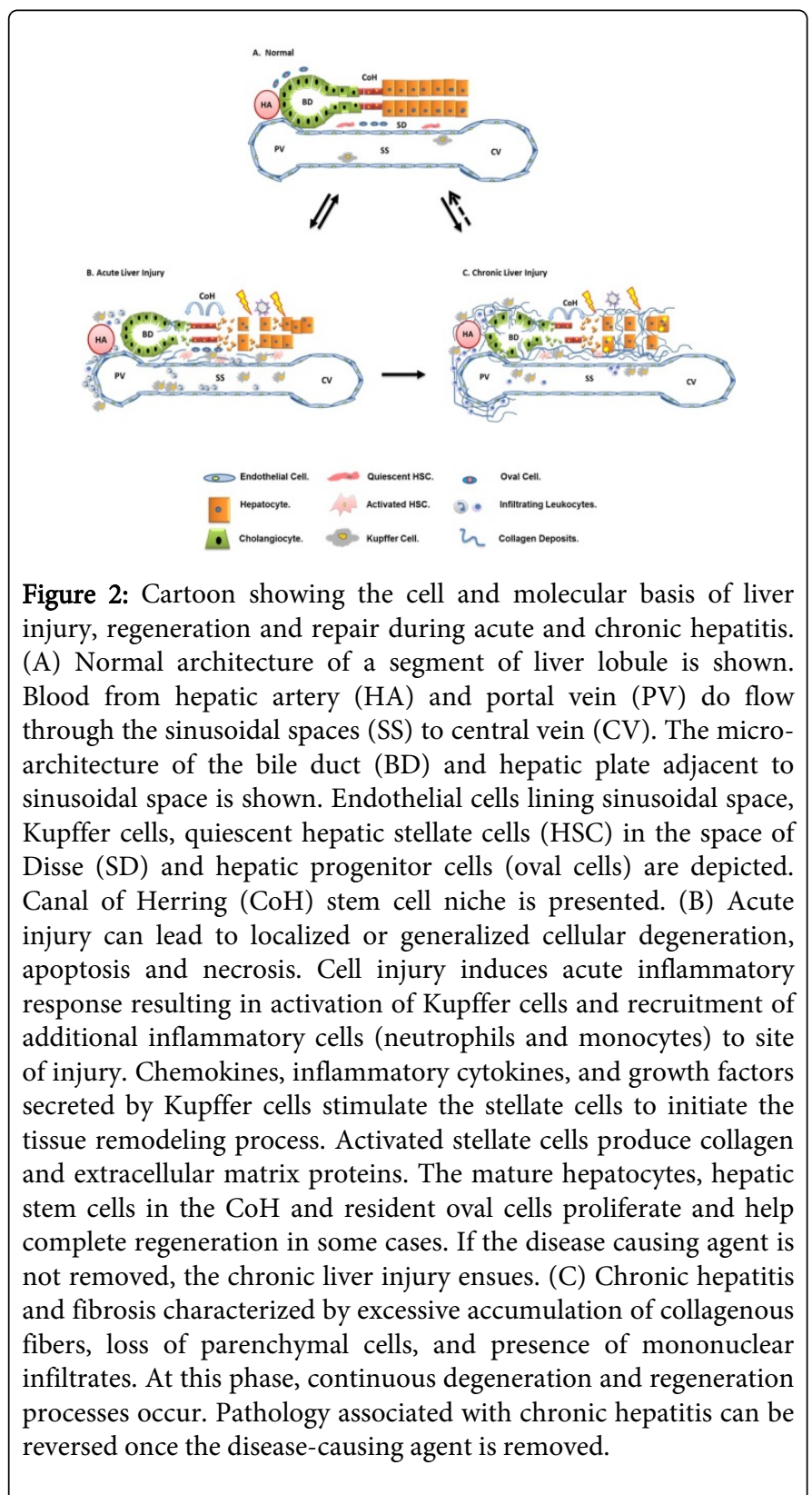


In the adult liver, bipotential progenitor/stem cells called oval cells have also been reported [33]. The oval cell population was first observed in the rat liver and has been shown to have originated in biliary ductules called the canals of Hering, which are known to compose the principal stem cell niche in adult livers [34-36]. Oval cells express cytokeratin 19, CD90/Thy-1, CD34, CD133 and c-kit markers which some are shared among the hematopoietic progenitor cell population [33,37]. Self-renewing liver stem cells marked by Wnt target gene Lgr5 have been described in murine liver [38]. Using the human embryonic stem cell (hESC) differentiation system, a novel hepatic progenitor cell population expressing the KDR (VEGFR2/ FLK-1) receptor was identified and subsequently verified in both human and mouse livers [39].

\section{Liver Injury, Repair and Regeneration Processes}

Throughout history, the liver has been known as the regenerative organ which dates back to Greek mythology with the well versed Blood of Prometheus. Nonetheless, its remarkable capacity for regeneration is best illustrated by recovery after partial hepatectomy. Restoration of the resected lobules has been attributed to the proliferative capacity of adult hepatocytes, which restore liver mass and function in a process called compensatory growth [40-42]. In these acute phases of injury, the hepatocytes are able to enter the cell cycle through a combinedparacrine cascade of induced cytokines and growth factors such as HGF, EGF, TGF- $\alpha$, TNF- $\alpha$, u-PA (plasminogen activator, urokinase) and IL-6, which are mainly generated by the surrounding nonparenchymal cells that constitute the liver[40,43-47]. Higher levels of HGF, CXCL12/SDF-1, and IGF-1 were present within the first 6 hours in the plasma of patients who underwent partial hepatectomy [48]. HGF and its receptor cMET are involved in embryonic liver development, hepatocyte differentiation and liver regeneration [49-52]. Beta-catenin signaling pathway has been shown to be critical for liver regeneration [53]. Recent evidence suggests that IL-22 and IL-17 secreting $\gamma \delta \mathrm{T}$ lymphocytes promote regeneration of liver [54]. The growth factors stimulate hepatocytes into a primed state of cell division (from G0 to G1) and proceed to enter the cell cycle from the dormant, quiescent state and proliferate. In mild injuries, replicating hepatocytes, biliary ductule cells and stromal cells regenerate the liver completely.

When the remarkable regenerative capacity of the hepatocytes in response to severe injury has been exhausted, the contribution of the bipotential progenitor cells, oval cells, becomes apparent. Biological factors IL-6, OSM, TGF- $\alpha$, HGF, and FGF have been shown to aid in the proliferation process. In acute or chronic liver injury, the cluster of proliferating liver progenitor cells can be observed histologically as ductular reactions [55,56]. During liver injury, progenitor cells from bone marrow and circulating blood can be attracted to site of injury. CXCL12 is involved in the attracting and homing of stem and progenitor cells through binding to the G-protein coupled receptor CXCR4 from circulation to the target organ or site of inflammation [57-60].

During persistent, long-term inflammation caused by chronic hepatitis $\mathrm{B} / \mathrm{C}$ viral infection, alcohol abuse and the massive accumulation of toxic chemical substances the compensatory liver regeneration process by the parenchyma becomes worn out and can result in the compensatory replacement of damaged liver cells with abnormal collagen-rich connective scar tissue, a process called fibrosis (Figure 2). Upon liver injury, the quiescent hepatic stellate cells undergo trans differentiation into activated myofibroblast-like cells [expressing $\alpha$-smooth muscle actin ( $\alpha$-SMA)] that play a key role in fibrogenesis $[30,61,62]$. TGF- $\beta$ secreted by inflammatory cells and Kupffer cells induce activated HSCs to produce collagen. In the cirrhotic liver, the extracellular matrix (ECM) component is remodeled into fibrillar type I collagen. The balance between the activities of ECM remodeling enzymes, liver interstitial collagenases, matrix metalloproteinases (MMPs) and tissue inhibitor of matrix metalloproteinases (TIMPs), controls the volume of deposited collagen [63]. During fibrosis, excessive intermolecular cross-linking of collagen molecules occurs, which has the effect of stabilizing the collagen deposits in the fibrotic lesions; thus, contributing to greater rigidity and proliferation of collagen-rich fibrotic scar tissue. As more of this scar tissue forms and accumulates, the normal architecture and function of the liver can be disrupted, which can result in irreversible scarring/regenerative nodule formation in what is called cirrhosis. Cirrhosis can cause hepatocellular carcinomas and also impair portal vein blood flow into the liver, resulting in the formation of portal hypertension and ascites. Hepatocytes and HSCs produce IGF-1 that increases liver regeneration after injury and reduces liver fibrogenesis [64]. The following section briefly describes viral hepatitis that shares many of the cellular and molecular aspects of acute and chronic inflammatory responses delineated earlier.

\section{Viral Hepatitis}

The hepatitis $\mathrm{C}(\mathrm{HCV})$ virus is a member of the Flaviviridae virus family, and is a small (about $50 \mathrm{~nm}$ in diameter), positive sense, enveloped, single-stranded RNA virus. HCV is transmitted by exposure to infected blood via sharing contaminated needle and blood transfusions. In around $80 \%$ of cases, HCV infection becomes chronic $[36,37]$. Upon exposure, HCV replicates mainly in hepatocytes and a small proportion in mononuclear cells, biliary epithelial cells and sinusoidal lining cells [65]. The body's immune response in the majority of patients (around $80 \%$ ) is ineffective in eliminating the virus, which leads to chronic inflammation of the liver. The inefficiency of the immune response to HCV has been linked to a weak $\mathrm{CD}^{+}$and $\mathrm{CD}^{+}{ }^{+}$-cell response during the acute phase likely due to the immuno-suppressive effects of hepatitis $\mathrm{C}$ viral factors such as core, NS3 and NS5A [32,34].

Hepatitis B Virus (HBV), a member of the Orthohepadnavirus genus of the virus family Hepadnaviridae, is an icosahedral, lipidenveloped virus containing a circular, partially double-stranded DNA genome (full-length negative strand and shortened positive strand) with a length of about 3,200 base pairs $[34,38]$. There are 8-9 distinct HBV genotypes that have been identified and labeled as genotypes A-I [38]. HBV is transmitted through contact with blood, semen, and other bodily fluids of an infected individual. The most common routes of infection are intravenous drug use, sexual contact, and perinatal transmission [38-41]. HBV, which targets hepatocytes, is known to be non-cytopathic. The main source of hepatocyte/liver tissue damage is the host immune response and the inflammation that results [34]. HBV infection can cause both acute and chronic infections. HBV infection in adults usually results in only acute hepatitis; while, perinatal HBV infection almost always results in chronic hepatitis. As with most other viruses, immunocompromised individuals are at higher risk for developing chronic hepatitis B [38-41].

Hepatitis A virus (HAV), a RNA virus (genus: Hepatovirus, Family: Picornaviridae), causes acute hepatitis and the infection is mostly selflimiting [66,67]. Hepatitis D virus (HDV) is a viroid-like agent consisting of a circular RNA genome. HDV replication takes place in 
cells already infected with HBV since HDV uses hepatitis B surface envelope proteins to package its RNA genome. Co-infection may result in fulminant hepatitis. In hepatitis $\mathrm{B}$ and $\mathrm{C}$, ductular reactions indicating proliferation of hepatic progenitor cells in the portal area have been reported $[55,68,69]$. In hepatitis $C$, cytokeratin 7 expressing progenitor cells in ductular reactions were correlated with fibrosis [68]. The viral-mediated biological effect on the progenitor cell population is unknown.

\section{Perspectives}

This review provides an in depth analysis of the key cell and molecular basis of the viral infection, inflammation, repair and regenerative processes of liver. New tools and technologies developed in the recent years usher an exciting era of investigation on stem cells and infectious diseases. Adult stem cells and progenitor cells are a very minor population in a tissue or organ, which poses the challenge of studying the pathophysiological effect of viral infection on stem cells. Recent discovery of induced pluripotent stem cells (iPSCs) provides an unlimited supply of defined organ-specific progenitor and mature cells which can be a very useful tool for modeling viral infections. Lineage tracing studies in animal models following viral infection can allow tracking of progenitor cell population in action during inflammatory and healing processes.

Upon injury activation of innate immune factors, DNA repair machinery, and inflammation pathways orchestrate the cell fate decision of apoptosis, cell survival, proliferation and differentiation to limit tissue or organ damage. Tissue remodeling and regenerative processes are greatly influenced by the severity and duration of exposure to the injurious stimuli. Massive tissue necrosis observed in diseases like fulminant hepatitis $\mathrm{C} / \mathrm{B}$ and influenza pneumonia can lead to organ failure without having sufficient time for the regenerative process to start. On the other end of the disease spectrum, chronic or repeated long-term exposure of tissues to injurious agents in conditions such as chronic hepatitis $\mathrm{C}$ can provide ample chances for tissue regeneration to take place. However, in chronic conditions, exhaustion of stem cells and loss of functional parenchymatous cells lead to fibrous scar tissue formation. In order to facilitate tissue repair or regeneration through stem cell therapy or allow body's natural ability to heal, the disease causing agent has to be removed. Combinatorial therapies consisting of direct acting anti-viral agents and organ-specific stem cells have the potential to greatly improve the clinical outcomes. Further investigation can help assess the stem cell role in degenerative viral diseases and identify a therapeutic window for cell therapy interventions.

\section{Acknowledgement}

We thank Jane Kang for editing this manuscript. We also thank members of the Arumugaswami laboratory for critical discussion and suggestions. Part of this work was funded by Cedars-Sinai Medical Center's Institutional Research Award and National Center for Advancing Translational Sciences, Grant UL1TR000124 to V.A.

\section{References}

1. Averhoff FM, Glass N, Holtzman D (2012) Global burden of hepatitis C: considerations for healthcare providers in the United States. Clin Infect Dis 55 Suppl 1: S10-15.

2. Alter MJ (2007) Epidemiology of hepatitis C virus infection. World J Gastroenterol 13: 2436-2441.
3. Ott JJ, Stevens GA, Groeger J, Wiersma ST (2012) Global epidemiology of hepatitis B virus infection: new estimates of age-specific HBsAg seroprevalence and endemicity. Vaccine 30: 2212-2219.

4. UNAIDS (2013) AIDS by the numbers.

5. Mao L, Yang Y, Qiu Y, Yang Y (2012) Annual economic impacts of seasonal influenza on US counties: spatial heterogeneity and patterns. Int J Health Geogr 11: 16.

6. Ploss A, Evans MJ, Gaysinskaya VA, Panis M, You H, et al. (2009) Human occludin is a hepatitis $\mathrm{C}$ virus entry factor required for infection of mouse cells. Nature 457: 882-886.

7. Weis W, Brown JH, Cusack S, Paulson JC, Skehel JJ, et al. (1988) Structure of the influenza virus haemagglutinin complexed with its receptor, sialic acid. Nature 333: 426-431.

8. Bergelson JM, Cunningham JA, Droguett G, Kurt-Jones EA, Krithivas A, et al. (1997) Isolation of a common receptor for Coxsackie B viruses and adenoviruses 2 and 5. Science 275: 1320-1323.

9. Tomko RP, Xu R, Philipson L (1997) HCAR and MCAR: the human and mouse cellular receptors for subgroup $\mathrm{C}$ adenoviruses and group $\mathrm{B}$ coxsackieviruses. Proc Natl Acad Sci U S A 94: 3352-3356.

10. Grove J, Marsh M (2011) The cell biology of receptor-mediated virus entry. J Cell Biol 195: 1071-1082.

11. Sugimoto N, Mitoma H, Kim T, Hanabuchi S, Liu YJ (2014) Helicase proteins DHX29 and RIG-I cosense cytosolic nucleic acids in the human airway system. Proc Natl Acad Sci U S A 111: 7747-7752.

12. Saito T, Owen DM, Jiang F, Marcotrigiano J, Gale M Jr (2008) Innate immunity induced by composition-dependent RIG-I recognition of hepatitis C virus RNA. Nature 454: 523-527.

13. Cotran RS, Kumar V, Collins T (1999) Acute and Chronic Inflammation In Robbins Pathologic Basis of Disease 6 edit., 50-88. W.B. Saunders Company, Philadelphia.

14. Aoki A, Ilahi MA, Koumans RJ, Burke JF (1968) Alterations in lymphatic flow caused by early acute bacterial inflammation. Surg Forum 19: 69-71.

15. Willoughby DA, Giroud JP (1969) The role of polymorphonuclear leucocytes in acute inflammation in agranulocytic rats. J Pathol 98: 53-60.

16. Willoughby DA, Coote E, Turk JL (1969) Complement in acute inflammation. J Pathol 97: 295-305.

17. Ryan GB, Majno G (1977) Acute inflammation. A review. Am J Pathol 86: $183-276$.

18. Lemon SM (1985) Type A viral hepatitis. New developments in an old disease. N Engl J Med 313: 1059-1067.

19. Medina RA, García-Sastre A (2011) Influenza A viruses: new research developments. Nat Rev Microbiol 9: 590-603.

20. Münz C, Lünemann JD, Getts MT, Miller SD (2009) Antiviral immune responses: triggers of or triggered by autoimmunity? Nat Rev Immunol 9: 246-258.

21. Jankosky C, Deussing E, Gibson RL, Haverkos HW (2012) Viruses and vitamin $\mathrm{D}$ in the etiology of type 1 diabetes mellitus and multiple sclerosis. Virus Res 163: 424-430.

22. Barker N (2014) Adult intestinal stem cells: critical drivers of epithelial homeostasis and regeneration. Nat Rev Mol Cell Biol 15: 19-33.

23. Martin P (1997) Wound healing--aiming for perfect skin regeneration. Science 276: 75-81.

24. Doan PL, Himburg HA, Helms K, Russell JL, Fixsen E, et al. (2013) Epidermal growth factor regulates hematopoietic regeneration after radiation injury. Nat Med 19: 295-304.

25. Carr ME, McKendrick GD, Spyridakis T (1976) The clinical features of infantile gastroenteritis due to rotavirus. Scand J Infect Dis 8: 241-243.

26. Snodgrass DR, Angus KW, Gray EW (1977) Rotavirus infection in lambs: pathogenesis and pathology. Arch Virol 55: 263-274.

27. Corcoran MS, van Well GT, van Loo IH (2014) Diagnosis of viral gastroenteritis in children: interpretation of real-time PCR results and relation to clinical symptoms. Eur J Clin Microbiol Infect Dis 33: 1663-1673. 
28. Kennedy JL, Turner RB, Braciale T, Heymann PW, Borish L (2012) Pathogenesis of rhinovirus infection. Curr Opin Virol 2: 287-293.

29. Robinson CH (1877) Cirrhosis of the Liver: Alcoholic Paralysis. Br Med J 1: 352-353.

30. Wang Y, Li J, Wang X, Sang M, Ho W (2013) Hepatic stellate cells, liver innate immunity, and hepatitis C virus. J Gastroenterol Hepatol 28 Suppl 1: 112-115.

31. Yokoi Y, Namihisa T, Kuroda H, Komatsu I, Miyazaki A, et al. (1984) Immunocytochemical detection of desmin in fat-storing cells (Ito cells). Hepatology 4: 709-714.

32. Gard AL, White FP, Dutton GR (1985) Extra-neural glial fibrillary acidic protein (GFAP) immunoreactivity in perisinusoidal stellate cells of rat liver. J Neuroimmunol 8: 359-375.

33. Masson NM, Currie IS, Terrace JD, Garden OJ, Parks RW, et al. (2006) Hepatic progenitor cells in human fetal liver express the oval cell marker Thy-1. Am J Physiol Gastrointest Liver Physiol 291: G45-54.

34. Petersen B, Shupe T (2008) Location is everything: the liver stem cell niche. Hepatology 47: 1810-1812.

35. Kordes C, Häussinger D (2013) Hepatic stem cell niches. J Clin Invest 123: 1874-1880.

36. Fausto N, Campbell JS (2003) The role of hepatocytes and oval cells in liver regeneration and repopulation. Mech Dev 120: 117-130.

37. Chen Q, Khoury M, Limmon G, Choolani M, Chan JK, et al. (2013) Human fetal hepatic progenitor cells are distinct from, but closely related to, hematopoietic stem/progenitor cells. Stem Cells 31: 1160-1169.

38. Huch M, Dorrell C, Boj SF, van Es JH, Li VS, et al. (2013) In vitro expansion of single Lgr5+ liver stem cells induced by Wnt-driven regeneration. Nature 494: 247-250.

39. Goldman O, Han S, Sourrisseau M, Dziedzic N, Hamou W, et al. (2013) KDR identifies a conserved human and murine hepatic progenitor and instructs early liver development. Cell Stem Cell 12: 748-760.

40. Michalopoulos GK (2010) Liver regeneration after partial hepatectomy: critical analysis of mechanistic dilemmas. Am J Pathol 176: 2-13.

41. Yokoyama ho, Wilson ME, Tsuboi KK, Stowell RE (1953) Regeneration of mouse liver after partial hepatectomy. Cancer Res 13: 80-85.

42. Widmann JJ, Fahimi HD (1975) Proliferation of mononuclear phagocytes (Kupffer cells) and endothelial cells in regenerating rat liver. A light and electron microscopic cytochemical study. Am J Pathol 80: 349-366.

43. Fausto N (2004) Liver regeneration and repair: hepatocytes, progenitor cells, and stem cells. Hepatology 39: 1477-1487.

44. Blindenbacher A, Wang X, Langer I, Savino R, Terracciano L, et al. (2003) Interleukin 6 is important for survival after partial hepatectomy in mice. Hepatology 38: 674-682.

45. Chaisson ML, Brooling JT, Ladiges W, Tsai S, Fausto N (2002) Hepatocyte-specific inhibition of NF-kappaB leads to apoptosis after TNF treatment, but not after partial hepatectomy. J Clin Invest 110: 193-202.

46. Weinstein M, Monga SP, Liu Y, Brodie SG, Tang Y, et al. (2001) Smad proteins and hepatocyte growth factor control parallel regulatory pathways that converge on betal-integrin to promote normal liver development. Mol Cell Biol 21: 5122-5131.

47. Bhanumathy CD, Tang Y, Monga SP, Katuri V, Cox JA, et al. (2002) Itih-4, a serine protease inhibitor regulated in interleukin-6-dependent liver formation: role in liver development and regeneration. Dev Dyn 223: 59-69.

48. Lehwald N, Duhme C, Wildner M, Kuhn S, Fürst G, et al. (2014) HGF and SDF-1-mediated mobilization of CD133+ BMSC for hepatic regeneration following extensive liver resection. Liver Int 34: 89-101.

49. Block GD, Locker J, Bowen WC, Petersen BE, Katyal S, et al. (1996) Population Expansion, Clonal Growth, and Specific Differentiation Patterns in Primary Cultures of Hepatocytes Induced by HGF/SF, EGFand TGFalpha in a Chemically Defined (HGM) Medium. The Journal of Cell Biology 132: 1133-1149.
50. Huh CG, Factor VM, Sánchez A, Uchida K, Conner EA, et al. (2004) Hepatocyte growth factor/c-met signaling pathway is required for efficient liver regeneration and repair. Proc Natl Acad Sci U S A 101: 4477-4482.

51. Ishikawa T, Factor VM, Marquardt JU, Raggi C, Seo D, et al. (2012) Hepatocyte growth factor/c-met signaling is required for stem-cellmediated liver regeneration in mice. Hepatology 55: 1215-1226.

52. Schmidt C, Bladt F, Goedecke S, Brinkmann V, Zschiesche W, et al. (1995) Scatter factor/hepatocyte growth factor is essential for liver development. Nature 373: 699-702.

53. Yang J, Mowry LE, Nejak-Bowen KN, Okabe H, Diegel CR, et al. (2014) $\hat{\mathrm{I}}^{2}$-catenin signaling in murine liver zonation and regeneration: a WntWnt situation! Hepatology 60: 964-976.

54. Rao R, Graffeo CS, Gulati R, Jamal M, Narayan S, et al. (2014) Interleukin 17-producing $\hat{\mathrm{I}}^{3} \hat{\mathrm{I}}^{\prime} \mathrm{T}$ cells promote hepatic regeneration in mice. Gastroenterology 147: 473-484.

55. Gouw AS, Clouston AD, Theise ND (2011) Ductular reactions in human liver: diversity at the interface. Hepatology 54: 1853-1863.

56. Williams MJ, Clouston AD, Forbes SJ (2014) Links between hepatic fibrosis, ductular reaction, and progenitor cell expansion. Gastroenterology 146: 349-356.

57. Baggiolini M (1998) Chemokines and leukocyte traffic. Nature 392: 565-568.

58. Aiuti A, Webb IJ, Bleul C, Springer T, Gutierrez-Ramos JC (1997) The chemokine SDF-1 is a chemoattractant for human CD34+ hematopoietic progenitor cells and provides a new mechanism to explain the mobilization of CD34+ progenitors to peripheral blood. J Exp Med 185: 111-120.

59. Kim CH, Broxmeyer HE (1999) Chemokines: signal lamps for trafficking of $\mathrm{T}$ and $\mathrm{B}$ cells for development and effector function. J Leukoc Biol 65: 6-15.

60. Rossi D, Zlotnik A (2000) The biology of chemokines and their receptors. Annu Rev Immunol 18: 217-242.

61. Ramadori G, Veit T, Schwögler S, Dienes HP, Knittel T, et al. (1990) Expression of the gene of the alpha-smooth muscle-actin isoform in rat liver and in rat fat-storing (ITO) cells. Virchows Arch B Cell Pathol Incl Mol Pathol 59: 349-357.

62. Carpino G, Morini S, Ginanni Corradini S, Franchitto A, Merli M, et al. (2005) Alpha-SMA expression in hepatic stellate cells and quantitative analysis of hepatic fibrosis in cirrhosis and in recurrent chronic hepatitis after liver transplantation. Dig Liver Dis 37: 349-356.

63. Kallis YN, Robson AJ, Fallowfield JA, Thomas HC, Alison MR, et al (2011) Remodelling of extracellular matrix is a requirement for the hepatic progenitor cell response. Gut 60: 525-533.

64. Sanz S, Pucilowska JB, Liu S, Rodríguez-Ortigosa CM, Lund PK, et al. (2005) Expression of insulin-like growth factor I by activated hepatic stellate cells reduces fibrogenesis and enhances regeneration after liver injury. Gut 54: 134-141.

65. Nouri-Aria KT, Sallie R, Mizokami M, Portmann BC, Williams R (1995) Intrahepatic expression of hepatitis $\mathrm{C}$ virus antigens in chronic liver disease. J Pathol 175: 77-83.

66. Gravelle CR, Hornbeck CL, Maynard JE, Schable CA, Cook EH, et al. (1975) Hepatitis A: report of a common-source outbreak with recovery of a possible etiologic agent. II. Laboratory studies. J Infect Dis 131: 167-171.

67. Feng Z, Hensley L, McKnight KL, Hu F, Madden V, et al. (2013) A pathogenic picornavirus acquires an envelope by hijacking cellular membranes. Nature 496: 367-371.

68. Clouston AD, Powell EE, Walsh MJ, Richardson MM, Demetris AJ, et al. (2005) Fibrosis correlates with a ductular reaction in hepatitis C: roles of impaired replication, progenitor cells and steatosis. Hepatology 41: 809-818.

69. Falkowski O, An HJ, Ianus IA, Chiriboga L, Yee H, et al. (2003) Regeneration of hepatocyte 'buds' in cirrhosis from intrabiliary stem cells. J Hepatol 39: 357-364. 